ACCepted for publication in the Astrophysical Journal

Preprint typeset using $\mathrm{LAT}_{\mathrm{E}} \mathrm{X}$ style emulateapj v. 11/10/09

\title{
GALAXY FORMATION WITH COLD GAS ACCRETION AND EVOLVING STELLAR INITIAL MASS FUNCTION
}

\author{
Xi KAnG ${ }^{1,2}$, W. P. $\operatorname{Lin}^{3}$, RAmin SkibBa ${ }^{2,4}$, D. N. Chen ${ }^{5}$ \\ 1 The Purple Mountain Observatory, 2 West Beijing Road, Nanjing 210008, China \\ 2 Max-Planck-Institute for Astronomy, Königstuhl 17, D-69117 Heidelberg, Germany \\ 3 Key Laboratory for Research in Galaxies and Cosmology, Shanghai Astronomy Observatory, Nandan Road 80, Shanghai, 200030, \\ China \\ 4 Steward Observatory, University of Arizona, 933 N.Cherry Avenue, Tucson, AZ 85721, USA and \\ 5 Beijing Planetarium, No 138 Xizhimenwai Street, Beijing 100044, China \\ Accepted for publication in the Astrophysical Journal
}

\begin{abstract}
The evolution of the galaxy stellar mass function is especially useful to test the current model of galaxy formation. Observational data have revealed a few inconsistencies with predictions from the $\Lambda$ CDM model. For example, most massive galaxies have already been observed at very high redshifts, and they have experienced only mild evolution since then. In conflict with this, semi-analytical models of galaxy formation predict an insufficient number of massive galaxies at high redshift and a rapid evolution between redshift 1 and 0 . In addition, there is a strong correlation between star formation rate and stellar mass for star-forming galaxies, which can be roughly reproduced with the model, but with a normalization that is too low at high redshift. Furthermore, the stellar mass density obtained from the integral of the cosmic star formation history is higher than the measured one by a factor of 2. In this paper, we study these issues using a semi-analytical model that includes: 1) cold gas accretion in massive halos at high redshift; 2) tidal stripping of stellar mass from satellite galaxies; and 3) an evolving stellar initial mass function (bottom-light) with a higher gas recycle fraction. Our results show that the combined effects from 1) and 2) can predict sufficiently massive galaxies at high redshifts and reproduce their mild evolution at low redshift, While the combined effects of 1) and 3) can reproduce the correlation between star formation rate and stellar mass for star-forming galaxies across wide range of redshifts. A bottom-light/top-heavy stellar IMF could partly resolve the conflict between the stellar mass density and cosmic star formation history.

Subject headings: galaxies: high-redshift — galaxies: mass function — galaxies: formation — galaxies: evolution — stars: mass function
\end{abstract}

\section{INTRODUCTION}

The current paradigm of structure formation based on the cold dark matter (CDM) model can explain the pattern of mass distribution on large scales (e.g., Springel et al. 2006). In this picture, structure ( consisting of dark matter halos) forms in a hierarchical manner such that small halos tend to form relatively early and subsequently merge to form large halos. Galaxies are expected to form at the center of dark matter halos via star formation in clouds of cooling gas (White \& Rees 1978). In the last few decades, semi-analytical models (SAMs) of galaxy formation, which combine the formation history of dark matter halos and physical descriptions of star formation and feedback mechanisms, have been established as useful tools for investigating the impacts of different physical processes on galaxy properties. Overall, SAMs can be constructed such that they agree with a wide rage of observations. In particular, with realistic models (including supernova and AGN feedback), the SAMs can match the faint and luminous end of luminosity functions of local galaxies well (see Baugh et al. 2007 for a review).

Recently, observations of galaxies at high- $z$ have revealed some issues that are difficult to explain with the model. Most importantly, the data suggest that the formation of galaxies is 'anti-hierarchical' (sometimes re-

kangxi@pmo.ac.cn ferred to as 'downsizing'; see Cowie et al. 1996). Most massive galaxies were already in place at very early epochs (e.g., Pozzetti et al. 2003), while low-mass galaxies were still forming with modest star formation rates. Measurements of the galaxy stellar mass function also show that massive galaxies evolve relatively little from $z=1$ to $z=0$, whereas the SAMs predict an insufficient number of massive galaxies at high- $z$ (e.g., Kitzbichler \& White 2007; Fontanot et al. 2009) and the predicted evolution of massive galaxies is too rapid compared to the observations (e.g., De Lucia et al. 2007) unless the disruptions of satellite galaxies are invoked (Monaco et al. 2006; Conroy et al. 2007; Somerville et al. 2008).

The absence of massive galaxies at early times in the SAMs is related to the modeling of gas cooling (e.g., Yoshida et al. 2002; Cattaneo et al. 2006). In most SAMs it is assumed that hot gas is isothermally distributed and cools down via radiative cooling, as described by the classical model of White \& Frenk (1991). However, it was recently found that gas cooling is dominated by cold accretion, which is efficient in halos with mass below $10^{12} M_{\odot}$, where the gas pressure is unable to support a stable shock (e.g., Kereš et a. 2005). In addition, cold accretions are also efficient in high- $z$ massive halos, as cold gas can flow along filaments (Birnboim \& Dekel 2003; Dekel et al. 2009).

Star-forming galaxies have a strong correlation between star formation rate and stellar mass (SFR- $M_{*}$ re- 
lation), and this relation evolves strongly with redshift (e.g., Brinchmann \& Ellis 2000; Brinchmann et al. 2004; Feulner et al. 2005; Daddi et al. 2007; Elbaz et al. 2007; Noeske et al. 2007; Zheng et al. 2007; Drory \& Alvarez 2008; Dunne et al. 2008). SAMs can approximately match the observed SFR- $M_{*}$ relation (Kitzbichler \& White 2007), but with a normalization that is too low at $z>0$ (Daddi et al. 2007; Elbaz et al. 2007). Khochfar \& Silk (2009) recently showed that the inclusion of cold accretion can boost the $\mathrm{SFR}-M_{*}$ relation to agree with the data at $z=2$.

The observed SFR- $M_{*}$ relation is dependent on the assumed stellar initial mass function (IMF). Davé (2008) proposed a stellar IMF, which is bottom-light compared to the Kroupa IMF (Kroupa 2001) at high- $z$, and they find that the observed normalization of SFR- $M_{*}$ relation can be reduced with a carefully determined stellar IMF. An evolving IMF is not a naive speculation, although its exact form is far from clear. There is theoretical and observational evidence for the stellar IMF at high- $z$ to be top-heavy or bottom-light (e.g., Larson 2005; van Dokkum 2008). Constraints on the stellar IMF can also be obtained from comparisons between the evolution of cosmic star formation rate and stellar mass density. For example, Wilkins et al. (2008) found that the stellar mass density derived from the cosmic star formation history is higher than the measured one by a factor of $\sim 2-3$ with the use of standard IMFs (Salpeter 1954; Kroupa 2001; Chabrier 2003). However, some have argued that this discrepancy may be due to a dust effect (Driver et al. 2007) and incompleteness in the measured stellar mass density (Reddy \& Steidel 2009). A top-heavy IMF is also favored in order to provide enough photons to ionize the universe at $z>7$ (e.g., Bunker et al. 2009).

In this paper, we use the model of Kang et al. (2005) to study the evolution of the stellar mass function, luminosity function, and SFR- $M_{*}$ relation. We modify the model to include cold gas accretion, and we use the evolving IMF proposed by van Dokkum (2008). In Section 2.2, we introduce the model and its modifications. In Section 3, we compare the predicted stellar mass and luminosity functions to the data. We discuss the SFR$M_{*}$ relation in Section 4 and present the evolution of star formation history in Section [5] We briefly conclude the paper in Section 6.

\section{SEMI-ANALYTICAL MODEL \\ 2.1. N-body Simulation}

We construct halo merger trees from a cosmological N-body simulation with cosmological parameters $\Omega_{m}=$ $0.24, \Omega_{\Lambda}=0.76$, and $\sigma_{8}=0.76$, which are similar to the WMAP3 cosmology results (Spergel et al. 2007). The simulation was made using Gadget-2 (Springel 2005) and follows $512^{3}$ particles of mass $5 \times 10^{8} M_{\odot} / h$ in a cube box of $100 \mathrm{Mpc} / \mathrm{h}$ on each side. We locate dark matter halos in the simulation using the standard friendsof-friends (FOF) method, and identify subhalos in the FOF halos. We then construct the subhalo merger trees and apply the physical model to describe star formation within these subhalos (see Kang et al. 2005 for details).

\subsection{Model for galaxy formation}

The model used in this paper is based on Kang et al. (2005; 2006; Kang 2009). Kang et al. (2005) show in detail how to graft the galaxy formation model onto the subhalo-resolved merger trees. In Kang et al. (2006), an AGN feedback model was included to suppress gas cooling in massive halos. Such a feedback mechanism is found to reproduce the luminosity of central cluster galaxies well (e.g., Croton et al. 2006; Bower et al. 2006; Somerville et al. 2008). The model is further improved by Kang (2009) to include a photo-ionization model to suppress the formation of very faint galaxies. Photoionization is essential to reproduce the luminosity function of Milky-Way satellites ( Macciò et al. 2009). We refer the reader to these papers for more details.

In the following, we will introduce the modifications to the previous version of the model, including a simple description of cold gas accretion, an evolving IMF, and a more realistic dust model to describe the extinction in galactic disks and starburst galaxies. We also follow Somerville et al. (2008) to model the stellar mass stripping of satellite galaxies.

- We include a simple description for cold gas accretion, following results from simulations. In previous models, gas is assumed to be shock-heated to the halo virial temperature and then cool down via radiation. The cooling rate is described by Equation 3-5 of Kang et al. (2005) and Equation 3 of Kang et al (2006). Here, we refer to this cooling description as hot-mode accretion. Kereš et al. (2005; 2008) have shown that cold-mode accretion is expected in halos of mass below $3-10 \times 10^{11} M_{\odot}$ (a threshold that depends on metallicity), as the gas is never heated to the halo virial temperature. Dekel \& Birnboim (2006; Dekel et al. 2009) have shown that even for massive halos $M>10^{12} M_{\odot}$ at high $-z$, cold streams still dominate, as the gas is infalling along filaments that are thin compared to the halo virial radius and hence avoids being shock heated. Both phases of gas accretion can occur simultaneously, and their fraction varies with halo mass and redshift (Ocvirk et al. 2008).

Dekel et al. (2009) have shown that the gas accretion is dominated by cold flows within halos below a critical mass, which can be described as $M_{c}=$ $M_{0} \max \left[1,10^{1.1\left(z-z_{c}\right)}\right]$. Depending on the metallicity of the gas , $M_{0}$ is about $\sim 3-20 \times 10^{11} M_{\odot}$, and $z_{c}$ is between $\sim 1.3-2$. Here we use $M_{0}=10^{12} M_{\odot}$ and $z_{c}=2.0$. All available gas in halos with mass below $M_{c}$ is assumed to be accreted into the central disk by the halo dynamical time-scale. For massive halos $\left(M>M_{c}\right)$, we continue to use hot-mode accretion.

In general, the cold accretion model allows more rapid gas accumulation in massive halos at high$z$. We will show in Section 3 that compared to the hot-mode accretion it produces more massive galaxies at $z>2$ and predicts a larger number of galaxies with high star formation rates.

- All previous SAMs use constant stellar IMFs. In this paper, we consider two possible stellar IMFs. The first is the still frequently used Chabrier IMF (Chabrier 2003, hereafter C03). The other is the evolving IMF proposed by van Dokkum (2008, 
hereafter VD08). Although there are no direct observational measurements of the stellar IMF at high- $z$, there is emerging evidence for an evolving IMF from both theoretical and observational arguments. Theoretically, Larson (2005; also Jappsen et al. 2005) suggests that the characteristic mass of star formation is related to the minimum temperature of molecular clouds, which increases with redshift due to heating from cosmic microwave radiation. Observationally, some have also favored an evolving stellar IMF (e.g., Lucatello et al. 2005; Stanway et al. 2005; Hopkins \& Beacom 2006; Tumlinson 2007; Wilkins et al. 2008; Elmegreen et al. 2008; Bunker et al. 2009). For example, Wilkins et al. (2008, also Davé 2008) have shown that a standard stellar IMF (Salpeter IMF or Chabrier IMF) produces a higher stellar mass density than the observed one.

Recently, van Dokkum (2008) studied the color and mass-to-light ratio of elliptical galaxies between $0<z<1$, and found that the data favor an IMF with fewer low-mass stars at $z \sim 4$. They used a modified Chabrier IMF to fit the IMF data of the Milky Way disk, globular cluster, submmgalaxies (see their Equation 18 and 20). For the VD08 IMF, its characteristic mass $m_{c}$ evolves with redshift, such that at $z=0$ it is $0.08 M_{\odot}(\mathrm{C} 03 \mathrm{IMF})$, but increases to $1 M_{\odot}$ at $z=5$. Thus, the VD08 IMF includes more high-mass stars at high redshifts.

Observationally, the star formation rates and stellar mass are both dependent on the assumed stellar IMF. Therefore, the data should be corrected if a different stellar IMF is used. For this purpose, we use the stellar population synthesis code of PEGASE.2 (Fioc \& Rocca-Volmerange 1997). We refer the reader to van Dokkum (2008) for details about how to correct the SFR and stellar mass. The SFR correction is relatively simple because it is only dependent on the number of massive stars. The stellar mass correction is not straightforward, as it is also dependent on the assumed star formation history.

An important effect from varying IMF is that the model parameter $R$, the gas recycle fraction from evolving stars, should also change. Following most SAMs, we calculate $R$ from a stellar population synthesis model, using the PEGASE.2. Wilkins et al. (2008) have shown that different models produce similar values of $R$. An IMF consisting of more high-mass stars will return more gas into the interstellar medium, and produce a lower stellar remnant mass. We found that higher $R$ will result in a higher normalization of the SFR-stellar mass correlation. Also higher $R$ is helpful to solve the discrepancy between the measured stellar mass density and that inferred from the cosmic star formation history.

- The tidal stripping of galaxy stellar mass is observed in the Milky Way (Ibata et al. 2001) as well as in galaxy clusters (e.g., Mihos et al. 2005; Zibetti et al. 2005). N-body simulation is a useful tool for studying this process, although currently there is a lack of detailed studies about how the stripping efficiency depends on the properties of the satellite galaxy and its host halo. Here we follow Somerville et al. (2008) by assuming that before merging with central galaxies, a fraction $f_{s}$ of the stellar mass of satellite galaxies is stripped and scattered into the intracluster region. We take their value of 0.4 for $f_{s}$, and we find that the intracluster light consists of about $8 \%$ of the total cluster light in our model, which is consistent with the results of Zibetti et al. (2005). Due to the difficult-to-measure low surface brightness objects, the measured fraction of intracluster light spans a wide range between $5-50 \%$ (see review by $\operatorname{Lin} \&$ Mohr 2004; Feldmeier et al. 2004).

- A dust extinction model similar to that of Guo \& White (2009) is used in this paper. Compared to the previous version of our model, it accounts for the dependence of optical depth on the cold gas fraction and metallicity. We apply this dust extinction model to galaxy disks. For galaxies experiencing recent starbursts, we use the dust model of Calzetti et al. (2000) instead, and adopt $E(B-V)$ as 0.3 , which is typical for dusty starburst galaxies (Calzetti et al. 2000; Poggianti \& Wu 2000). In the resulting model, we find that at $z=0$ most starburst galaxies reside in halos with mass around $10^{12} M_{\odot}$.

\section{STELLAR MASS AND LUMINOSITY FUNCTIONS}

In this section, we compare the predicted stellar mass function (SMF ) and rest-frame $B$ - and $K$-band luminosity functions to the observational measurements. The SMF has been extensively measured in recent decades using multi-wavelength photometry data from large area surveys. The uncertainties in the measured stellar mass result from the adopted stellar IMF, dust correction, metallicity, and assumed star formation history, among which the stellar IMF contributes the dominant systematic error between different data sets. It is simple to correct the stellar mass between the often used Salpeter, Kroupa (2001) and C03 IMFs, which are assumed to be independent of galaxy mass and redshift (Bell et al. 2003). As the characteristic mass of the VD08 IMF evolves with time, the correction does depend on galaxy mass and redshift (Marchesini et al. 2009), and determining these dependences is beyond the scope of this paper. For our comparison of galaxy SMFs, we continue to use the C03 IMF and focus on the effects of cold accretion and tidal stripping on the model predictions.

In Fig. 1, we plot the data from Drory et al. (2005, triangles), Fontana et al. (2006, circles) and Marchesini et al. (2009, squares) at $z>0$, and the data at $z \sim 0$ are from Bell et al. (2003, triangles) and Cole et al. (2001, squares). They are all converted to the C03 IMF. The best fit to the data at $z=0$ by Cole et al. (2001) is duplicated in each plot by the gray solid line, and the black and color lines are our model predictions.

Firstly, it is clear that while the data are consistent at the massive end, the measurements differ by a factor of $\sim 2-3$ for galaxies with mass below $10^{10.5} M_{\odot}$. This is mainly due to an incomplete analysis of the errors. Most work consider only the Poisson errors or errors from pho- 


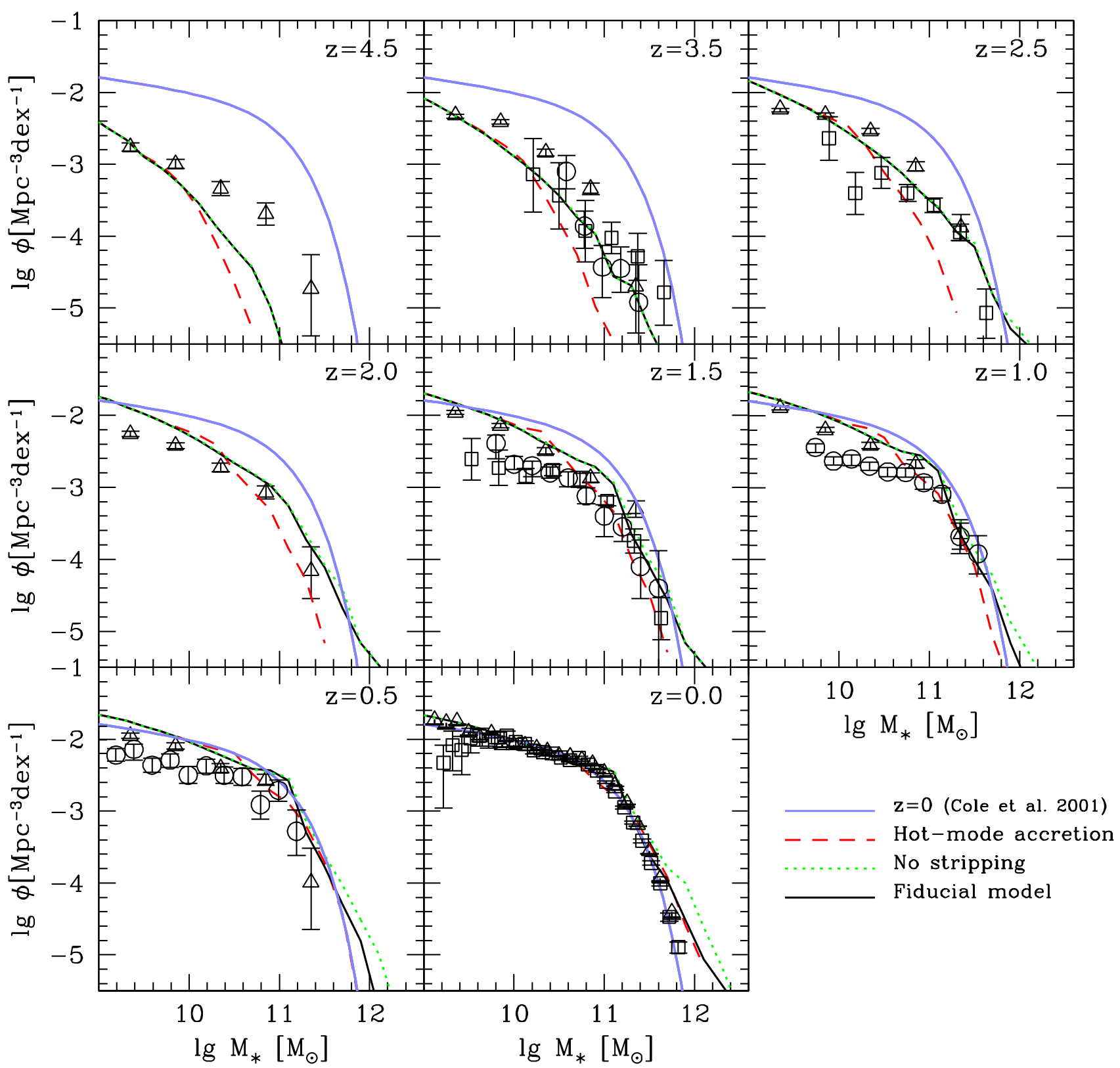

FIG. 1. - The evolution of galaxy stellar mass functions. The data points at $\mathrm{z}=0$ are taken from Cole et al. (2001, squares), Bell et al. (2003, triangles). The high-z data are taken from Drory et al. (2005, triangles), Fontana et al. (2006, circles), and Marchesini et al. (2009, squares). The best fit to the local SMF by Cole et al. is duplicated as gray line in each panel. The red-dashed, green-dotted and black-solid lines are our model predictions under different assumptions, see the main text. Note that here all data are transferred into the Chabrier 2003 IMF.

tometric redshift uncertainties. In addition, field-to-filed variations are also a significant source of error (Marchesini et al. 2009). The green dotted lines show the model prediction without tidal stripping. As one can see, between $z=1$ and $z=0$, this model predicts a stronger evolution at the massive end than indicated by the data (Also see Somerville et al. 2008). The red dashed lines show the prediction for hot-mode accretion, and we find that it produces a good match to the local stellar mass function, but predicts too few massive galaxies at $z>2$. Our fiducial model, which includes both tidal stripping and cold gas accretion, produces a fair match to the stellar mass function from $z=4$ to $z=0$. Still, we find that all of the SAMs predict an overabundance of galaxies with mass around $10^{11} M_{\odot}$ at $z \sim 1$. Nonetheless, the inclusion of cold gas accretion is helpful to produce more massive galaxies at high redshift, while the tidal stripping can reproduce the mild evolution of the stellar mass function at the massive end from $z=1$ to $z=0$.

Although the galaxy stellar mass function is important to constrain the SAMs, determining the galaxy stellar mass involves uncertainties from the modeling. Marchesini et al. (2009) have analyzed the varies effects, such as dust, metallicity, and stellar population synthesis, on the measured stellar mass. For example, they have found that the stellar mass derived from the Maraston (2005) model is lower by 0.2 dex compared to that obtained using the Bruzual \& Charlot (2003) model, and it is weakly dependent on mass and redshift. In this case, we argue that the stellar mass correction has no significant effects 


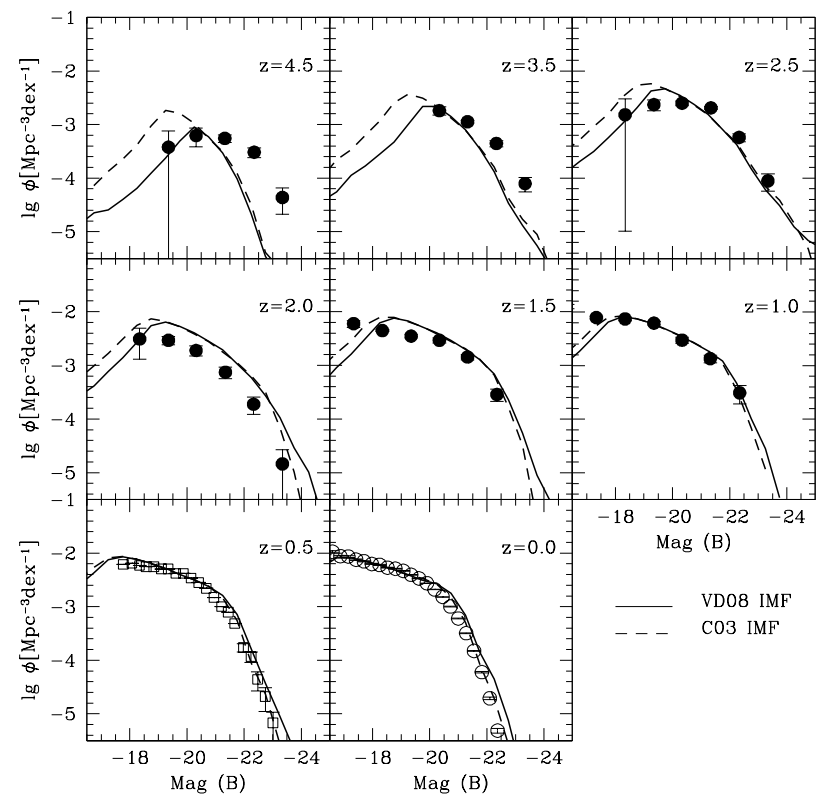

FIG. 2.- The evolution of rest-frame B band (AB magnitude) luminosity functions. Data points are taken from Norberg et al. (2002, $\mathrm{z}=0$ ), Wolf et al. (2003, squares at $\mathrm{z}=0.5$ ), and Gabasch et al. (2004, solid circles at $z>0.5$ ). Solid and dashed lines are our model predictions for two stellar IMFs. Here we also use dust extinction to young star-burst galaxies, see the text.

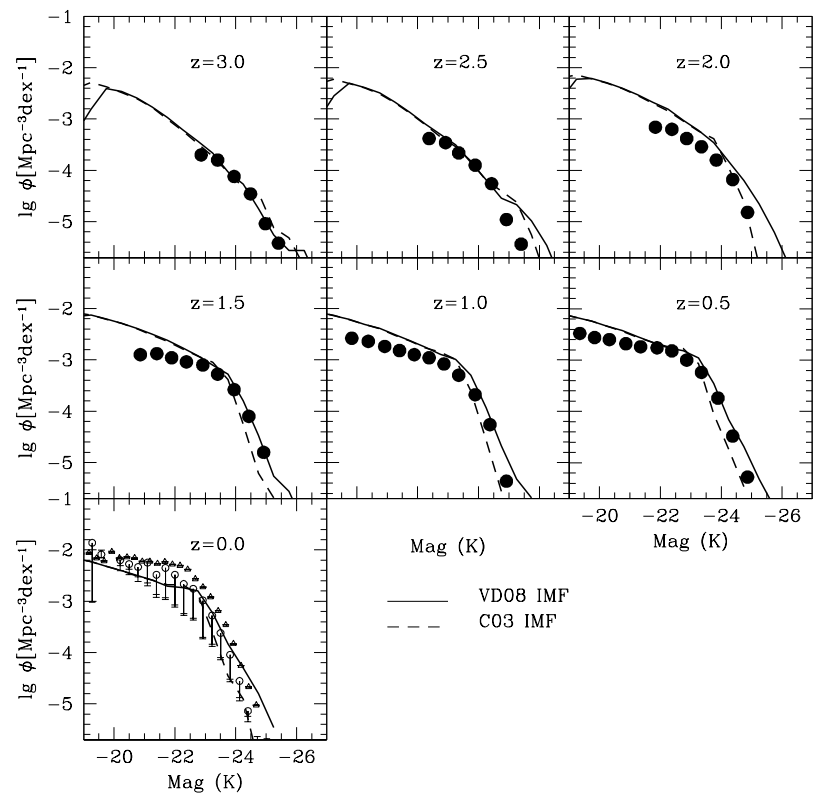

Fig. 3.- As in Fig.2, but for the rest-frame K band luminosity functions. Data points are taken from Cole et al. (2001,triangles at $\mathrm{z}=0$ ), Huang et al. (2003, circles at $\mathrm{z}=0$ ), and Cirasuolo et al. (2007, solid circles). Note that the rest-K band luminosity functions are shown up to $\mathrm{z}=3$, lower than the B-band LFs in Fig.2

on our results, as we have normalized our model parameters using the local stellar mass function. It is the evolution of the galaxy stellar mass function that puts strong constraints on our model parameters.

We show the evolution of the rest-frame $B$ - and $K$ band luminosity functions (LFs) in Fig. 2 and Fig. 3 , respectively. The $B$-band $\mathrm{LF}$ is more sensitive to the recent star formation activity of galaxies, rather than the total stellar mass indicated by the $K$-band LF. Measurements of LFs at both bands can set constraints on the star formation history of galaxies. Observational data have improved rapidly over recent years. However, due to the magnitude limit and survey volume, the LFs at both the faint and luminous ends are not well constrained, but in general, all of these measured LFs are consistent with each other.

The $B$-band data are taken from Norberg et al. (2002), Wolf et al. (2003), Gabasch et al (2004). The $K$-band data are taken from Cole et al. (2001), Huang et al. (2003), and Cirasuolo et al. (2007). Our model predictions are shown as the solid and dashed lines for the VD08 and C03 IMFs, respectively. Overall we find good agreement between the model predictions and the data in both $B$ - and $K$ - bands. The agreement at high redshifts is encouraging as previous models (e.g., Kitzbichler \& White 2007; Bower et al. 2006) have under-predicted 


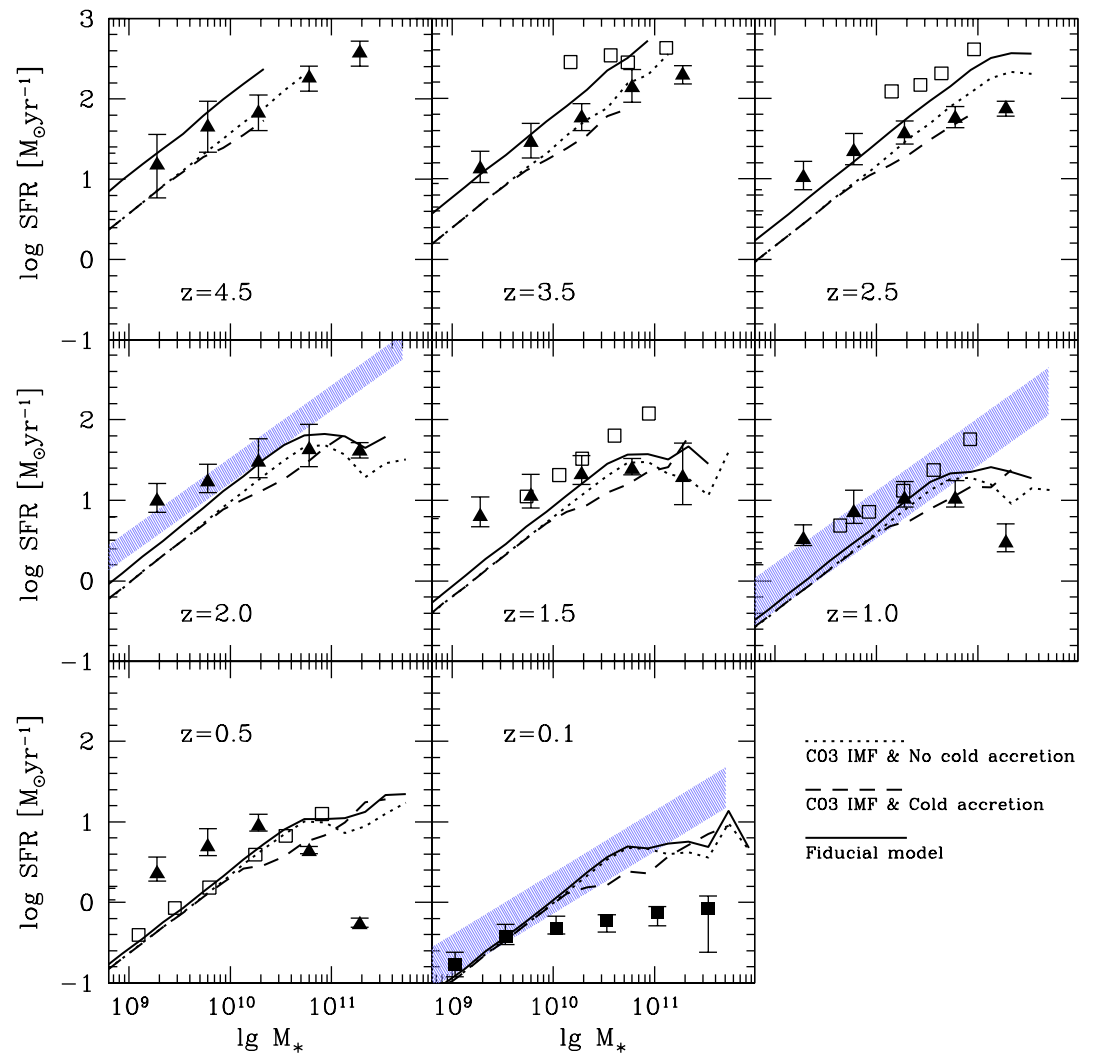

FIG. 4. - The star formation rate - stellar mass relation from $z=4.5$ to $z=0$. Data points are taken from Drory \& Alvarez (2008, triangles), Dunne et al. (2009, empty squares), and Chen et al. (2009, solid squares). Shade area are best fits (with $1 \sigma$ variance) by Daddi et al. (2007) and Elbaz et al. (2007) to the data at $z=2,1,0$. Our model predictions are shown as lines with model parameters indicated in the plot.

the abundance of bright galaxies in $K$-band at high redshifts. As stated previously, this is mainly due to the implementation of cold accretion in the model. Note that the $B$-band LFs drop off at the faint end at high redshift, and this is due to the resolution of our simulation, in which only halos with mass larger than $5 \times 10^{9} M_{\odot} / h$ are included.

We also find that the model predictions only slightly depend on the choice of IMF. This is unexpected, because the VD08 IMF contains more high-mass stars. However, as shown by van Dokkum (2008) and Marchesini et al. (2009), although there are more massive stars in the VD08 IMF, the number of characteristic stars that dominate the optical light, also decreases with increasing redshift. As a result, the net effects on the luminosity are modest.

\section{THE STAR FORMATION RATE - STELLAR MASS RELATION}

We investigate the SFR- $M_{*}$ relation in this section. A direct comparison between the model and the observational data is difficult, because of the various selection effects in the data. Firstly, the tight correlation between SFR and $M_{*}$ is observed only for star-forming galaxies, and there lacks of a simple and robust way to select star-forming galaxies in the model to match the various observational samples. Secondly, due to survey limits, many low-SFR (or passive) galaxies are not included in observational samples, which may give rise to a biased SFR- $M_{*}$ relation. However, given the tightness of the SFR- $M_{*}$ relation and its small scatter, the missing galaxies can not contribute significantly above the SFR detection limit (Davé et al. 2008).

We select all the model galaxies and plot their average SFR in Fig. 4. The data plotted are Drory \& Alvarez 2008; triangles), Dunne et al. (2009; open squares), and Chen et al. (2008; filled squares). The shaded area show the best fits (with $1 \sigma$ variance) to the data at $z=2,1,0$ quoted by Daddi et al. (2007) and Elbaz et al (2007). It is important to note that the Chen et al (2008) and Drory \& Alvarez (2008) data are the average SFR of all galaxies in their samples, while the other data are only for the main sequences of active star-forming galaxies or sBzK galaxies. This gives rise to the different behavior of the SFR- $M_{*}$ relation for massive galaxies, where the average SFR of all galaxies departs from the sBzK result because more massive and passive galaxies become dominant at lower redshift. For less massive galaxies, we can see that the data agree with each other within a factor of $\sim 2$.

Our model predictions are shown by the solid, dashed and dotted lines in Fig. 4. Our fiducial model, with cold accretion and an evolving IMF, can reproduce the evolution of the correlation between SFR and $M_{*}$ fairly well. Although our predictions do not agree with all of the measurements, they are within the range spanned by different data sets. For massive galaxies, our model predictions are in better agreement with Drory \& Alvarez (2008), because both are the average SFR of all galaxies. At lower redshift $(z<1)$, our SFR- $M_{*}$ relation has a higher tail than the data at the massive end. In general, our predicted SFR- $M_{*}$ relation is a significant improvement over previous results from SAMs (Croton 


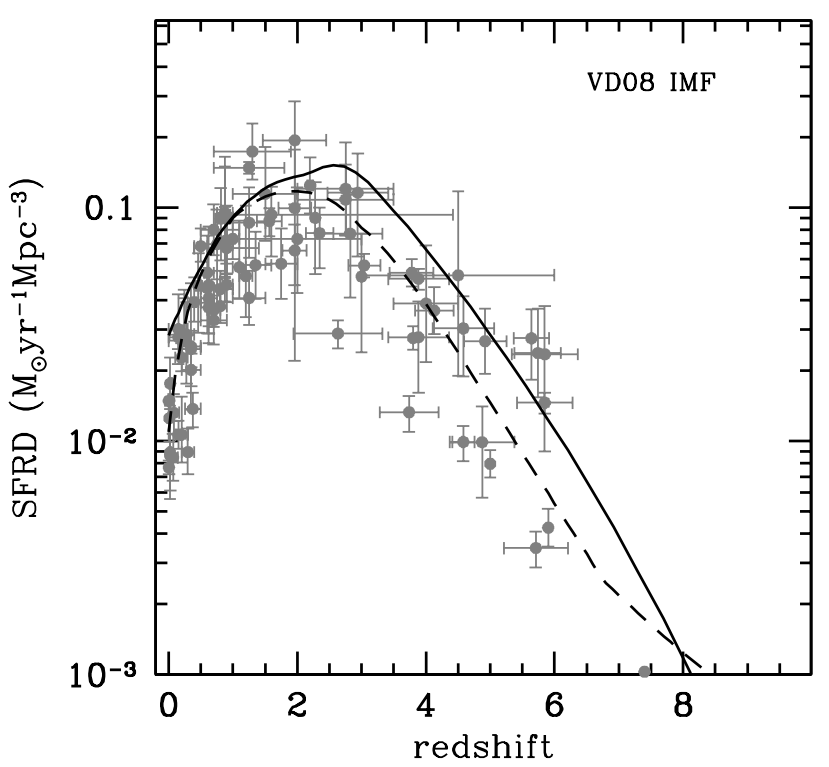

Fig. 5.- The cosmic star formation rate history. The gray points are taken from the compilation of observational data by Hopkins \& Beacom (2006), and the dashed line is the best fit to the data which are all corrected to the VD08 IMF. Our model prediction is plotted as the solid line.

et al. 2006; Kitzbichler \& White 2007), which have produced SFR- $M_{*}$ relations that are too low compared to the observations at $z=2$ and $z=1$. Recently Fontanot et al. (2009) have also examined the SFR- $M_{*}$ relation, but their predictions are still lower than the observations. In the following, we will investigate possible contributions to the boost of the SFR- $M_{*}$ relation in our model vis-á-vis other models.

For the model with the traditional hot-mode cooling and C03 IMF, the prediction is in agreement with the data at $z<1$, but is lower than the measurements at $z=2$. This is similar to the results of Kitzbichler \& White (2007) and Fontanot et al. (2009). In contrast, when cold accretion is included, the model predicts more galaxies with high star formation rates, so that the SFR$M_{*}$ relation is in better agreement for $M_{*}>10^{10} M_{\odot}$, but it is still lower that the data at $z>2$.

The normalization of the predicted SFR- $M_{*}$ relation is also boosted in our fiducial model by the VD08 IMF. This boost is larger at higher redshift, but is independent of galaxy mass. In the model with the VD08 IMF, at high redshift there are more massive stars that return more stellar winds to the interstellar medium and thus decrease the stellar remnant mass. For example, at $z=4$, the stellar remnant at age of $0.5 \mathrm{Gyr}$ is $28 \%$ of the initial formed mass with the VD08 IMF, but it is $63 \%$ for the C03 IMF. Thus for a given star formation rate history, the stellar mass is lower for the VD08 IMF, which significantly boosts the normalization of SFR- $M_{*}$ relation. We find that the $\mathrm{SFR}-M_{*}$ relation increases by $\approx 60 \%$ at $z=2$.

Finally, we note that these results depend strongly on the gas recycle fraction $R$. As noted previously, $R$ is calculated from stellar population synthesis, which involves uncertainties from the modeling of stellar winds and stellar evolution tracks. More strong constraints could be put on the IMF with more precise modeling of stellar winds and supernova feedback.

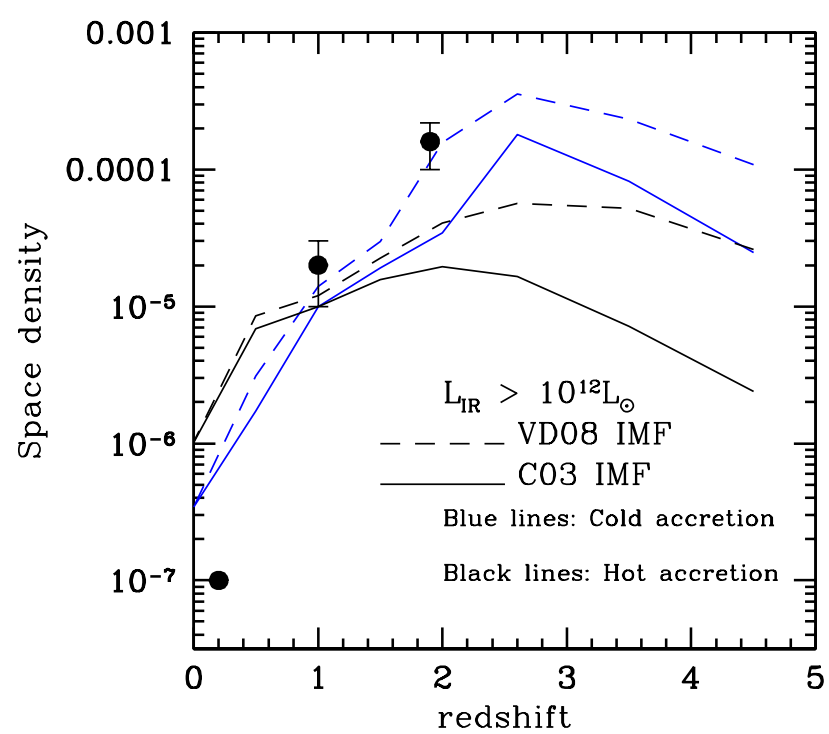

FIG. 6. - The space density of ULIRGs with $L_{I R}>10^{12} M_{\odot}$. The implied star formation rate for these objects is $S F R>$ $120 M_{\odot} / y r$ for the Chabrier $2003 \mathrm{IMF}$, but only $84 M_{\odot} / y r$ for the VD08 IMF at $\mathrm{z}=2$, which is bottom-light than the C03 IMF. The data point at $\mathrm{z}=2$ implies that both cold accretion and bottomlight (or top-heavy) IMF are needed to produce enough ULIRGs.

\section{COSMIC STAR FORMATION HISTORY}

Many recent observations have been dedicated to measuring the cosmic star formation history (e.g., Madau et al. 1996; Giavalisco et al. 2004; Bouwens et al. 2006). It has been found that the star formation rate density (SFRD) at redshift $1-2$ is higher than that of the local universe by an order of magnitude. The precise location of the peak of the SFRD is not yet clear, but it may be beyond $z \sim 2$ (Steidel et al. 1999). With more data from submm galaxies and Lyman break galaxies, the SFRD can be measured out to the very early universe $(z \sim 7)$. For galaxy formation models, it is important to understand which mechanism drives the rapid increase of SFRD at $z>2-3$, and the steep decrease at $z<1$.

As our model parameters are normalized by the local stellar mass function, we can make predictions for the history of the SFRD. In Fig. 5, we compare the prediction of our fiducial model to observations at $0<z<6$. The data are taken from the compilation of observational data by Hopkins \& Beacom (2006, and see references therein), and we add the measurements of Reddy \& Steidel (2008, pentagon points) and Bouwens et al. (2007, circles). All of the data and their best fit from Hopkins \& Beacom (dashed line) are converted to the VD08 IMF. We find that our model reproduces the overall evolution of the SFRD quite well, over a wide range of redshifts. At $z>3$ the data are not yet well constrained, although we expect that new data in the future will contribute additional constraints on the models.

Although our model predicts the total SFRD approximately, it is interesting to determine whether it also predicts a sufficient number of galaxies with very high star formation rates. The observations of ultra-luminous infrared galaxies (ULIRGs) indicate that they are possibly galaxies experiencing strong starbursts with dust obscuration (Rieke \& Lebofsky 1979). Daddi et al. (2007) found that the predicted number of intensely starforming galaxies in a SAM (Kitzbichler \& White 2007) 


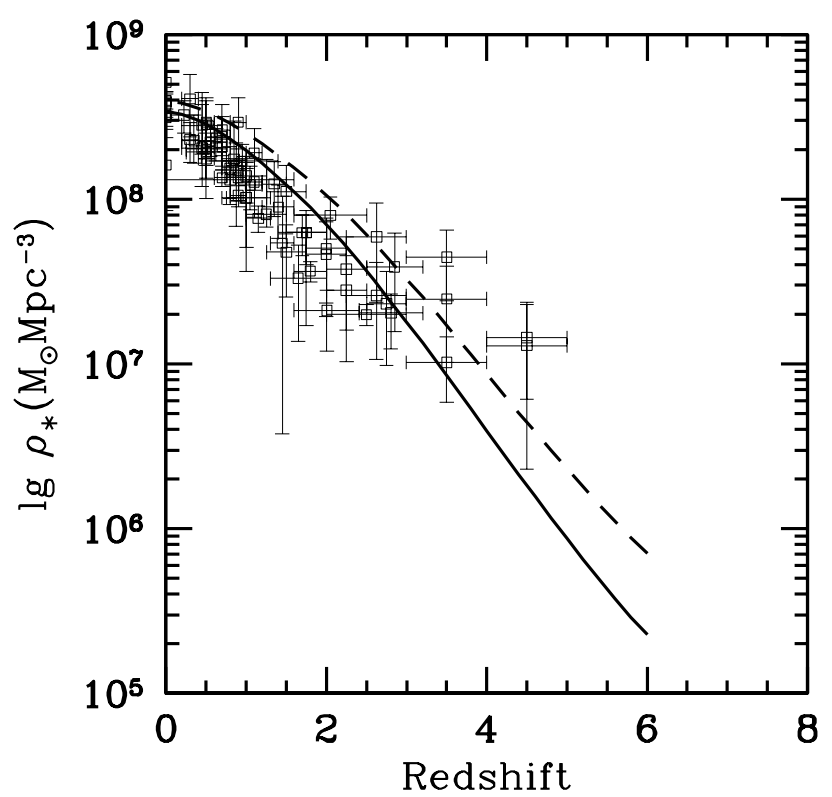

Fig. 7.- The evolution of stellar mass density. Data points are taken from the compilation by Wilkins et al. (2008). The lines are integrated stellar mass density from the cosmic star formation rate history (dashed line in Fig 5 . Different gas recycle fraction, $R$, are used for the two lines. For the dashed line, $R$ is taken from the C03 IMF, while the solid line uses $R$ from the VD08 IMF. We can find that better agreement is obtained if the stellar remnant mass is lower, which is the case with a bottom-light (or top-heavy) IMF.

is lower than the data at $z=1$ and 2 by an order of magnitude. Recently Khochfar \& Silk (2009) showed that the inclusion of cold accretion in their model produces enough ULIRGs.

In Fig. 6, we show our model predictions, and we compare them to the data from Sander et al. $(2003, z=0)$ and Daddi et al. (2007, $z>0)$. The blue and black lines show the model predictions with cold-mode and hot-mode accretion, respectively. We again consider two possible IMFs (C03 IMF: solid lines; VD08 IMF: dashed lines). The ULIRGs with $L>10^{12} L_{\odot}$ have star formation rates larger than $120 M_{\odot} / y r$ for the C03 IMF (for the VD08 IMF, the implied SFR at $z=2$ is $\left.84 M_{\odot} / y r\right)$.

As can be seen from the figure, both cold accretion and an evolving IMF are required for the model to be reconciled with the data. At $z=1$, the model cannot be constrained well as cold accretion is no longer very important and the VD08 IMF is similar to the C03 IMF. We note that data at $z>3$ contribute strong constraints on the model, and additional constraints will be obtained from observations from future high- $z$ submm galaxy surveys.

Finally, we compare the observed stellar mass density history with that expected from the integral of cosmic star formation history in Fig. 7. The integrated stellar mass is simply calculated as $\int S F R(t)(1-R) d t$, where $R$ is the fraction of gas returned to the inter-stellar medium by stellar winds and supernovae, which is dependent on the IMF and stellar ages. The data points in the figure show the measured stellar mass density compiled by Wilkins et al. (2008), and the lines are inferred from the cosmic star formation history (the same as the dashed line in Fig. (5), but with different parameter $R$.

Some have found that the inferred stellar mass density from the cosmic star formation history is higher than the measured one at $1<z<3$ with either a Salpeter IMF or Kroupa (2001) IMF (e.g., Wilkins et al. 2008). Our fiducial model's result (solid line) uses the VD08 IMF, and we also show the results with $R$ taken from the $\mathrm{C} 03$ IMF. We find that the VD08 IMF yields better agreement with the data, and this is partly due to the fact that the stellar remnant mass is lower with this IMF (i.e., higher $R)$.

\section{CONCLUSIONS}

In this paper, we use a semi-analytical model of galaxy formation to study the evolution of the galaxy stellar mass function, $B$ - and $K$-band luminosity functions, the star formation rate-stellar mass relation, and the cosmic star formation rate history. We modify our previous model to include cold gas accretion, an evolving stellar IMF, and stellar stripping from satellites. We focus on the impacts of these effects and assumptions on the predictions of the model, and we obtain the following results:

- Cold accretion in massive halos at high redshifts is crucial to produce a sufficient number of massive galaxies at $z>2$, in order to reconcile the model with the data. By accounting for cold accretion, as well as stellar stripping from satellite galaxies, our model can reproduce the mild evolution of the stellar mass function from $z=1$ to $z=0$. This resolves the problems of previous semi-analytical models, which have predicted too few massive galaxies at high redshift and too strong evolution at low redshift.

- The predicted star formation rate - stellar mass relation in previous semi-analytical models is too low at $z>1$. We show that such a correlation can be predicted from a SAM, and the normalization can be boosted to match the data if an evolving IMF is adopted. The main cause of the higher normalization is the larger number of high-mass stars in our adopted IMF (van Dokkum 2008), which produces a lower stellar remnant mass, due to stronger stellar winds and supernovae ejecta. We find that at $z=2$ the specific star formation rate is increased by $60 \%$ compared to a model with a Chabrier (2003) IMF.

- Our model is capable of reproducing the evolution of the cosmic star formation rate. An additional constraint can be placed on the model by using the number density of ULIRGs, which have relatively high star formation rates. We find that the combined effect from cold accretion and a bottom-light (or top-heavy) IMF can reproduce the number density of ULIRGs. The contradiction between the measured stellar mass density and the integrated one from the cosmic star formation rate can be resolved by using an IMF containing more high-mass stars.

In summary, we argue that there are currently no severe conflicts between the CDM model and galaxy observations. We can now explain issues that were previously considered to be major problems: the rapid formation of massive galaxies at high redshift and their mild evolution at low redshift; the high amplitude of the observed SFR- $M_{*}$ relation; and the apparent discrepancy between 
the evolving star formation rate density and stellar mass density. These discrepancies can be resolved in the context of galaxy formation based on the CDM theory.

Finally, we note that there are some uncertainties regarding our model assumptions. First, although the tidal stripping of satellite is crucial to stop the rapid growth of massive central galaxies, its efficiency should be studied in detail by more realistic simulations (e.g., Puchwein et al. 2010). Second, both the SFR-stellar mass relation and evolving stellar mass density are dependent on the gas recycle fraction, $R$, which in principle should be determined by the stellar IMF. Larger $R$ will decrease the stellar remnant mass and increase the normalization of the predicted SFR-stellar mass relation. Due to the uncertainties of modeling $R$ from stellar winds and evolution tracks, the favored bottom-light (or top-heavy) IMF deserves further investigation.

\section{ACKNOWLEDGMENTS}

We are grateful to XianZhong Zheng, Frank C. van den Bosch for enlightening discussions. We thank the referee for suggestions and comments to strengthen the presentation of our paper. The simulation was done at Shanghai Supercomputer center by the support of Chinese National 863 project (No. 2006AA01A125). $\mathrm{XK}$ is supported by the One Hundred Talents project of the Chinese Academy of Science. WPL acknowledge the supports from Chinese National 973 project (No. 2007CB815401), NSFC project (No. 10873027, $10821302,10533030)$ and the Knowledge innovation Program of the Chinese Academy of Sciences (grant KJCX2YW-T05).

\section{REFERENCES}

Baugh C. M., 2006, Rep. Prog. Phys., 69, 3101

Bell E. F., McIntosh D. H., Katz N., Weinberg M. D., 2003, ApJS, 149, 289

Birnboim, Y., Dekel, A., 2003, MNRAS, 345, 349

Bouwens R. J., Illingworth G. D., Franx M., Ford H., 2007, ApJ, 670,928

Bouwens R. J., Illingworth G. D., Blakeslee J. P., Frankx M., 2006, ApJ, 653, 53

Bower R. G., Benson A. J., Malbon R., Helly J. C., Frenk C. S., Baugh C. M., Cole S., Lacey C. G., 2006, MNRAS, 370, 645

Brichmann J., Ellis R. S., 2000, ApJ, 536, L77

Brinchmann J., Charlot S., White S. D. M., Tremonti C., Kauffmann G., Heckman T., Brinkmann J., 2004, MNRAS, 351, 1151

Bruzual G., Charlot S., 2003, MNRAS, 344, 1000

Bunker, A., et al. 2009, arXiv:0909.2255

Calzetti D., Armus L., Bohlin R., Kinney A. L., Koornneef J., Storchi-Bergmann T., 2000, ApJ, 533, 682

Cattaneo A., et al., 2007, MNRAS, 377, 63

Chabrier G., 2003, PASP, 115, 763

Chen Y-M., Wild V., Kauffmann G., Blaizot J., Davis M., Noeske K., Wang J-M., Willmer C., 2009, MNRAS, 393, 406

Cirasuolo M., et al., 2007, MNRAS, 380, 585

Cole S., et al., 2001, MNRAS, 326, 255

Conroy C., Wechsler R. H., Kravtsov A. V., 2007, ApJ, 668, 826

Cowie L. L., Songaila A., Hu E. M., Cohen J. G., 1996, AJ, 112, 839

Croton D. J., et al., 2006, MNRAS, 365, 11

Daddi E., et al., 2007, ApJ, 670, 156

Davé R., 2008, MNRAS, 385, 147

De Lucia G., Blaizot J., 2007, MNRAS, 375, 2

Dekel A., Birnboim Y., 2006, MNRAS, 368, 2

Dekel A., et al., 2009, Nature, 457, 451

Driver S. P., Popescu C. C., Tuffs R. J., Liske J., Graham A. W., Allen P. D., de Propris R., 2007, MNRAS, 379, 1022

Drory N., Salvato M., Gabasch A., Bender R., Hopp U., Feulner G., Pannella M., 2005, ApJ, 619, L131

Drory N., Alvarez M., 2008, ApJ, 680, 41

Dunne L., et al., 2009, MNRAS, 394, 3

Elbaz D., et al., 2007, A\&A, 468, 33

Elmegreen B. G., Klessen R. S., Wilson C. D., 2008, ApJ, 681, 365

Feldmeier J. J., Mihos J. C., Morrison H. L., Harding P., Kaib N., Dubinski J., 2004, ApJ, 609, 617

Feulner G., Gabasch A., Salvato M., Drory N., Hopp U., Bender R., 2005, ApJ, 633, L9

Fioc M., Rocca-Volmerange B., 1997, A\&A, 326, 950

Fontana A., et al., 2006, A\&A, 459, 745

Fontanot F., De Lucia G., Monaco P., Somerville, R., Santini P., MNRAS, 397, 1776

Gabasch A., et al. 2004, A\&A, 421, 41

Giavalisco ,.., et al., 2004, ApJ, 600,103

Glazebrook K., et al., 2004, Nature, 430, 181

Guo Q.,White S.D.M., 2009, MNRAS, 396, 39

Hopkins A. M., Beacom J. F., 2006, ApJ, 651, 142
Huang J. S., Glazebrook K., Cowie L. L., Tinney C., 2003, ApJ, 584,203

Ibata R, Irwin M., Lewis G., Ferguson A. M., Tanvir N., 2001, Nature, 412,49

Jappsen A.-K., Klessen R. S., Larson R. B., Li Y., Mac Low M.-M., 2005, A\&A, 435, 611

Kang X., Jing Y.P., Mo H.J., Börner G., 2005, ApJ, 631, 21

Kang X., Jing Y.P, Silk J., 2006, ApJ, 648, 820

Kang X., 2009, IAU Symposium, 254, 32P

Kereš D., Katz N., Weinberg D. H., Davé R., 2005, MNRAS, 363, 2

Kereš D., Katz N., Fardal M., Davé R., Weinberg D. H., 2009, MNRAS, 395, 160

Khochfar S., Silk J., 2009, ApJ, 700, L21

Kitzbichler M. G., White S. D. M., 2007, MNRAS, 376, 2

Kroupa P., 2001, MNRAS, 322, 231

Larson R. B., 2005, MNRAS, 359, 211

Lucatello S., Gratton R. G., Beers T. C., Carretta E., 2005, ApJL, 625, 833

Macciò A. V., Kang X., Fontanot F., Somerville R., Koposov S E., Manaco P, 2009, arXiv:0903.4681, MNRAS in press

Madau P., Ferguson H. C., Dickinson M. E., Giavalisco M., Steidel C. C., Fruchter A., 1996, MNRAS, 283, 1388

Maraston C., 2005, MNRAS, 362, 799

Marchesini D., van Dokkum P. G., Forster Schreiber N. M., Franx M., Labbe I., Wuyts S., 2009, ApJ, 701, 1765

Mihos J. C., Harding P., Feldmeier J., Morrison H., 2005, ApJ, 631, L41

Monaco P., Murante G., Borgani S., Fontanot F., 2006, ApJ, 652, L89

Noeske K. G., et al., 2007, ApJ, 660, L43

Norberg P. et al., 2002, MNRAS, 336, 907

Ocvirk P., Pichon C., Teyssier R., 2008, MNRAS, 390, 1326

Poggianti B., Wu H., 2000, ApJ, 529, 157

Pozzetti L., et al. 2003, A\&A, 402, 837

Puchwein E., Springel V., Sijacki D., Dolag K., arXiv:1001.3018

Reddy N. A., Steidel C. C., 2009, ApJ, 692, 778

Rieke G. H., Lebofsky M. J., 1979, ARA\&A, 17, 477

Salpeter E. E., 1955, ApJ, 121, 161

Sanders D. B., Mazzarella J. M., Kim D.-C., et al. 2003, AJ, 126, 1607

Somerville R., Hopkins P. F., Cox T. J., Robertson B. E.,

Herquist L., 2008, MNRAS, 391, 481

Spergel D. N., et al., 2007, ApJS, 170, 377

Springel V., 2005, MNRAS, 364, 1105

Springel V., Frenk C. S., White S. D. M., 2006, Nature, 440, 1137

Stanway E. R., McMahon R. G., Bunker A. J., 2005, MNRAS, 359,1184

Steidel C. C., Adelberger K. L., Giavalisco M., Dickinson M.,

Pettini M., 1999, ApJ, 519, 1

Tumlinson J., 2007, ApJ, 665, 136

van Dokkum P, 2008, ApJ, 674, 29

White S. D. M., Rees M. J., 1978, MNRAS, 183, 341

White S. D. M., Frenk C. S., 1991, ApJ, 379, 52 
Wilkins S. M, Trentham N., Hopkins A.M., 2008, MNRAS, 385, 687

Wolf C., Meisenheimer K., Rix H-W., Borch A., Dye S., Kleinheinrich M., 2003, A\&A, 401, 73

Yoshida N., Stoehr F., Springel V., White S. D. M., 2002, MNRAS, 335, 762
Zheng X. Z., Bell E. F., Papovich C., Wolf C., Meisenheimer K., Rix H.-W., Rieke G. H., Somerville R., 2007, ApJ, 661, L41

Zibetti S., White S. D. M., Schneider D. P., Brinkmann J., 2005, MNRAS, 358, 949 